\title{
Norois
}

Environnement, aménagement, société

245 | 2017

Adapter les territoires aux changements climatiques : transition urbanistique et aménagement de l'espace

\section{Note de recherche. L'adaptation, un concept systémique pour mieux panser les changements climatiques}

Adaptation: a systemic concept to heal climate change

\section{Guillaume Simonet}

\section{OpenEdition}

\section{Journals}

Édition électronique

URL : http://journals.openedition.org/norois/6252

DOI : $10.4000 /$ norois. 6252

ISBN : 78-2-7535-7465-6

ISSN : $1760-8546$

Éditeur

Presses universitaires de Rennes

\section{Édition imprimée}

Date de publication : 31 décembre 2017

Pagination : 113-125

ISBN : 978-2-7535-7456-4

ISSN : 0029-182X

Référence électronique

Guillaume Simonet, « Note de recherche. L'adaptation, un concept systémique pour mieux panser les changements climatiques », Norois [En ligne], 245 | 2017, mis en ligne le 31 décembre 2019, consulté le 05 janvier 2021. URL : http://journals.openedition.org/norois/6252 ; DOI : https://doi.org/10.4000/ norois. 6252 


Presses
Universitaires
de Rennes

\title{
Note de recherche \\ L'adaptation, un concept systémique pour mieux «panser» les changements climatiques

\author{
Research note-Adaptation: A Systemic Concept to Heal Climate Change
}

\section{Guillaume Simonet}

\begin{abstract}
Chercheur associé au Centre interdisciplinaire d'études urbaines, Laboratoire interdisciplinaire solidarités, sociétés, territoires UMR5193, université Toulouse Jean-Jaurès, Maison de la Recherche - 5, allée Antonio-Machado, 31058 Toulouse Cedex 9, France. (simonet.guillaume@ymail.com)
\end{abstract}

Résumé : L'élaboration d'une action publique territoriale en matière d'adaptation aux changements climatiques rencontre d'importants défis, parmi lequel celui de traduire en actions un concept riche en interprétations aussi divergentes les unes que les autres. En s'appuyant sur les résultats du projet ABSTRACT-colurba (2014-2015) mené auprès de dix collectivités françaises, l'article discute la difficulté d'élaborer puis de traduire sur le terrain des mesures dites « d'adaptation aux changements climatiques ». Parmi les facteurs expliquant cet état de faits, il est souligné l'inadéquation entre la construction théorique de la notion d'adaptation et la complexité d'une réalité interreliée auxquels les acteurs sont quotidiennement confrontés. Afin de contourner ces verrous et faciliter la mise en pratique d'actions en réponse aux changements climatiques, l'article conclut que la recherche d'une définition précise d'un terme aussi vaste que l'adaptation n'aide pas la mise en place d'une action publique territoriale climatique, laquelle gagnerait à être abordée de manière davantage systémique autour des enjeux environnementaux.

Abstract: The development of a territorial public action in the field of adaptation to climate change is facing important challenges. One of them is to translate the concept of adaptation into action because of its various interpretations. Based on the results of the ABSTRACTcolurba project (2014-2015) in ten French communities, the article discusses the difficulty of developing field measures called "adaptation to climate change". One of the elements explaining these facts is the mismatch between the theoretical construction of the notion of adaptation and the interrelated complex reality to which the actors are daily confronted to. In order to overcome these obstacles and facilitate the implementation of actions in response to climate change, the article concludes that the search for a precise definition of a term as vast as adaptation does not help the territorial public action establishment, which would better benefit from a systemic approach that would internalize the environmental issues.

Mots clés : changement climatique, développement local, durabilité, réification, sémantique, territorialité régionale

Keywords: climate change, local development, sustainability, reification, semantic, regional territoriality 


\section{INTRODUCTION : LES CONSTATS DE LA DIFFICULTÉ DE TRADUIRE L'ADAPTATION EN ACTIONS}

S'inscrivant dans un contexte imbriqué d'évolutions multiples (démographiques, socioéconomiques, environnementales, énergétiques) à l'échelle aussi bien globale que locale, l'accélération des changements climatiques est en cours. Dès lors, les effets d'une laborieuse réduction des émissions de gaz à effet de serre (GES) (ou mitigation), appréciables uniquement sur le long terme, rendent inéluctables l'ajustement et la transformation des systèmes en place en vue de réduire l'apport au phénomène et l'ampleur des impacts issus de tels bouleversements (Füssel, 2007). Ainsi, face à cet état de faits, l'adaptation interpelle nos sociétés à double titre. En premier lieu par la modification des territoires (aménagements, formes urbaines, technologies) afin de faire face aux impacts (directs et indirects) climatiques. Et d'autre part, par une modification des comportements des populations et des pratiques des activités, notamment en vue d'accélérer la transition énergétique vers une société sobre en carbone. Toutefois, la traduction opérationnelle d'actions se réclamant de l'adaptation aux changements climatiques se heurte à de nombreuses « barrières » significatives aux origines mal comprises et multiples (Jones et al., 2014), dont l'étude constitue un thème de recherche émergent (Barnett, 2010). Parmi ces barrières, celles reliées aux dynamiques organisationnelles entourant les prises de décision sont pointées comme un frein majeur dans les pays développés malgré le peu d'études sur le sujet (Naess et al., 2005; Grothmann et Patt, 2005). Et bien que l'adaptation soit considérée comme un processus social dynamique, les dimensions humaines et sociales ont jusque-là été peu approfondies alors qu'elles constituent le gros des obstacles en matière d'efficacité des mécanismes décisionnels (Huq et Reid, 2004 ; Adger et al., 2007). Certains auteurs soulignent l'importance du hiatus temporel entre le long terme (impacts climatiques) et le court terme (dynamiques politiques, prises de décision, roulement du personnel) (Biesbroek et al., 2013), difficultés exacerbées pour des collectivités aux ressources, à la volonté politique ou à l'expertise limitées (Crabbé et Robin, 2006). D’autres barrières ne sont pas directement liées à l'adaptation et se retrouvent dans toutes problématiques environnementales complexes ou lors d'implantation de politiques publiques, mais force est de constater que les changements climatiques renforcent des situations déjà problématiques (Moser et Ekstrom, 2010). Parmi ces freins institutionnels, plusieurs études pointent les barrières cognitives comme contraintes importantes au niveau des décideurs, soulignant le rôle prépondérant joué par la complexité des systèmes humains dans le succès ou l'échec d'élaboration et de mise en oeuvre de politiques d'adaptation (Lammel et al., 2012; Lorenzoni et al., 2007). Par exemple, le déni fait partie d'une panoplie d'attitudes aux origines cognitives, mentales et psychologiques complexes dont les influences sur les politiques ne sont pas assez considérées (Grothmann et Patt, 2005 ; Gifford, 2008). De même, les représentations sociales, l'acquisition des connaissances, la mémoire, l'attitude, les émotions ou la manière de percevoir les risques occupent une place prépondérante dans les recherches traitant des facteurs d'ordre cognitif à l'origine d'influences significatives sur la prise de décision dans le domaine des changements climatiques (Leiserowitz, 2006; Reser et al., 2011). Dans cette lignée, une des barrières principales à la mise en pratique d'une action collective en matière d'adaptation se situe dans la difficulté de saisir la signification (matérielle, conceptuelle) d'une "adaptation ", laissant place à une panoplie d'interprétations auprès des acteurs impliqués (Simonet, 2011 ; Simonet et Leseur, 2015). Dès lors, dans quelle mesure la variabilité des interprétations des acteurs influe sur les mécanismes décisionnels en amont des actions mises en place à l'échelle des collectivités? Doit-on se référer à une définition particulière de l'adaptation, et laquelle? Quels types d'activités faut-il y voir? Quelles ressources une collectivité locale doit déployer afin de contourner cette difficulté et mieux répondre aux injonctions de s'adapter aux changements climatiques?

La note propose de discuter ces points en s'appuyant sur les résultats du projet de recherche ABSTRACT-colurba (2014-2015) portant sur une analyse comparative de dix plans climat français. Après avoir brièvement rappelé l'essor du thème de l'adaptation aussi bien dans l'action publique locale que dans la recherche scientifique, l'article s'interroge sur les raisons des différences d'interprétations entre les institutions responsables des cadrages 
théoriques et prescriptifs et les acteurs locaux responsables de la mise en pratique à l'échelle de leur collectivité. Plusieurs hypothèses sont émises parmi lesquelles celle de l'influence des communications émises par le Groupe intergouvernemental des experts sur l'évolution du climat (GIEC), celle de l'historique conceptuel de l'adaptation et la manière de percevoir les changements climatiques. L'article conclut sur le fait que mieux penser l'adaptation permettrait de mieux panser les changements climatiques.

\section{Cadrage théorique et PRESCRIPTIF VS MISE EN PRATIQUE}

\section{L'essor de l'adaptation dans la recherche et les politiques locales}

Historiquement, c'est d'abord à travers la science du climat que l'adaptation aux changements climatiques retient l'attention scientifique, notamment en développant des modèles prospectifs de plus en plus affinés sur l'évolution du système climatique dans l'objectif de mieux cerner ses impacts futurs sur les systèmes naturels et humains. Cependant, du fait de l'impossibilité de prendre en compte de nombreuses variables locales, ces extrapolations ont difficilement été intégrables dans les politiques publiques locales (Dessai et Hulme, 2004). Les sciences humaines et sociales se sont donc, dans un deuxième temps, intéressées aux facteurs territoriaux déterminant l'habileté à s'adapter, développant une conception de l'adaptation qui prend en compte la vulnérabilité des éco-socio-systèmes (Füssel, 2007). Par la suite, la recherche sur l'adaptation a continué à s'interroger sur l'influence des caractéristiques sociales et politiques, d'idéologies, de contextes géographiques et d'articulation de plusieurs niveaux de gouvernance entre le global et le local dans les actions à mettre en place (Magnan et al., 2009). Aujourd'hui, la littérature scientifique sur l'adaptation aux changements climatiques est florissante et certains auteurs n'hésitent plus à y voir une véritable science du fait de ses caractéristiques spécifiques en termes de décloisonnement disciplinaire, de vision systémique ou d'intégration de la pensée complexe (Simonet, 2014).

En termes de cadrage institutionnel, l'adaptation aux changements climatiques intégra tout d'abord l'ordre du jour des négociations internationales une fois la responsabilité collective mise sur la table par les pays en développement (vulnérables) visà-vis des pays développés (historiquement émetteurs de GES) (Burton et al., 2002). Par la suite, les politiques publiques climatiques portant sur l'adaptation fleurirent à l'échelle nationale, puis au niveau local, soutenues par les urgences régulièrement lancées par les rapports du GIEC (Van Aalst et al., 2008). En France, à la suite de l'adhésion à la CCNUCC (1992) et du premier Programme national de lutte contre le changement climatique (2000), le premier Plan Climat vit le jour en 2004 dans lequel figurait l'adaptation parmi les huit principaux points (Aykut et Dahan, 2015). Une stratégie nationale d'adaptation au changement climatique fut publiée en 2007 avant de laisser sa place en 2011 au Plan national d'adaptation au changement climatique (PNACC), dont la seconde version révisée sortira en 2018. Localement, l'agglomération grenobloise fut la première à lancer un Plan Climat Énergie Territorial (PCET) en 2005 (Bertrand et Larrue, 2007). La Loi Grenelle II de 2010 portant engagement national pour l'environnement cristallise l'obligation pour les collectivités de se doter d'un PCET (devenu PC-Air-ET depuis la loi sur la Transition Énergétique de 2015) incluant un volet adaptation. En 2016, en application de la nouvelle organisation territoriale (loi NOTRe) et de la mise en place des nouvelles Régions, les conseils régionaux sont amenés à fusionner les anciens schémas régionaux en un Schéma régional d'aménagement, de développement durable et d'égalité des territoires (SRADDET), lequel document prescriptif contribue à définir les orientations en matière de réduction des émissions de GES et d'adaptation. Enfin, divers outils de planification intègrent l'adaptation aux changements climatiques à l'échelle de l'intercommunalité ou de la commune tels que les Schémas de Cohérence Territoriale (SCoT), les Plans locaux d'urbanisme (PLU) ainsi que les outils attenants à la gestion du risque (Plan communal de sauvegarde [PCS], Document d'Information Communal sur les Risques Majeurs [DICRIM]) ou à la Gestion des milieux aquatiques et la prévention des inondations (GEMAPI). 


\section{Un cadrage théorique axé sur l'ajustement}

Face aux changements climatiques, deux réponses furent prônées par le GIEC, instance onusienne scientifique de référence en matière de changements climatiques : la mitigation (ou réduction, ou atténuation en français) et l'adaptation. Dans cette conception, il s'agit d'engager des actions de réduction de gaz à effet de serre (GES) (mitigation) distinctes d'actions de protection face aux aléas météorologiques (adaptation). Cette dichotomie «mitigation-adaptation » présente donc l'adaptation comme le terme choisi pour caractériser la réponse qui s'attache à la gestion des impacts des changements climatiques sur les territoires, les populations et les activités. C'est sous cette définition que la recherche scientifique portant sur l'adaptation se diffusa au point qu'aujourd'hui, Basset et Fogelman (2013) estiment que plus de $70 \%$ de la littérature scientifique spécialisée sur le sujet mettent en avant cette dimension protection, appelée dans le reste de l'article « dimension ajustement». Cette définition situant l'adaptation dans le registre de la protection aux aléas météorologiques fut également largement reprise dans les cadrages prescriptifs des institutions publiques. En effet, que cela soit aussi bien dans les réglementations, dans les préconisations des guides pratiques ou dans certains discours ${ }^{1}$, l'adaptation fut rapidement assimilée à la "résilience », terme pour lequel on assiste à un véritable engouement (Quenault, 2013). Une des raisons tient du fait que la résilience bénéficie d'une terminologie à connotation positive, contrairement à l'adaptation (Simonet et Fatorić, 2015). Mais le concept de résilience, perçu comme capable de répondre au défi de la complexité, permet également de remobiliser les recherches associées à la géographie des risques et des catastrophes naturelles, et donc de traiter les changements climatiques au même titre qu'une problématique de gestion des risques (Pigeon, 2012). In fine, ces exposés encouragent de traduire l'adaptation en actions d'aménagement du territoire dans l'objectif de renforcer les systèmes en place pour se protéger des aléas climatiques.

1. «Des progrès considérables pewvent être réalisés grâce à l'océan dans les deux dimensions de sa relation au climat : l'atténuation et l'adaptation résiliente. " discours prononcé par Ségolène Royal, ministre de l'Environnement au moment de la COP21, 2 décembre 2015.
Traduites sur le terrain par les acteurs locaux, l'adaptation se matérialise par des réponses palliatives, souvent confinée à une liste d'actions pragmatiques mobilisant des savoir-faire situés dans la lignée du solutionnisme technique en place depuis plusieurs décennies (Reghezza-Zitt et Rufat, 2015). Cette vision technologiste et ingénieuriale s'effectue sans remise en cause de l'ordre social et politique établi, sans que ne soient discutés les liens entre la distribution des ressources, la pauvreté et les pouvoirs, et se développe au détriment d'une adaptation plus sociétale fondée sur les principes de solidarité et d'éthique, condition critique d'un développement urbain durable (Quenault, 2013; Basset et Fogelman, 2013). Pourtant, cette dimension « ajustement », nommée telle quelle dans le reste du texte, n'est qu'une définition partielle de ce que recouvre l'adaptation dans sa richesse conceptuelle, notion appropriée par de multiples disciplines notamment parce qu'elle interroge la manière dont doivent répondre les systèmes face aux changements de son environnement dont sa survie dépend.

\section{Présentation du projet ABSTRACT-COLURBA (2014-2015)}

C'est dans ce contexte de recherche que le projet ABSTRACT-colurba (Analyse des Barrières et leviers à la mise en place de STRatégies d'adaptation aux Changements climaTiques - le cas des collectivités urbaines) fut mené. Financée par l'Agence de l'environnement et de la maîtrise de l'énergie (ADEME) et l'Agence française de développement (AFD), la recherche fut portée par l'Institute for Climate Economics (I4CE) durant deux années (2014-2015). Son objectif principal fut d'explorer les barrières et leviers opérant sur les mécanismes décisionnels et les dynamiques organisationnelles sous-jacents à l'élaboration et la mise en œuvre de stratégies d'adaptation aux changements climatiques à l'échelle locale. Pour ce faire, le projet s'est appuyé sur une enquête sociologique de terrain auprès de dix collectivités territoriales françaises invitées à participer à l'étude selon trois critères : 1) l'engagement actif d'un Plan Climat Énergie Territorial (PCET) ; 2) des collectivités de taille « moyenne » (dite « intermédiaire » et à rayonnement régional); 3) des cas d'études représentant une diversité d'enjeux (milieux physiques, socioé- 
conomiques). Les collectivités sélectionnées furent : la Ville d'Annecy, la Communauté Urbaine de Dunkerque, Cergy-Pontoise Agglomération, la Ville de Sète, la Communauté d'Agglomération Royan Atlantique, Agen Agglomération, la Communauté Intercommunale Réunion Est, Territoire Côte Ouest (Réunion), la Communauté d'Agglomération du Centre de la Martinique et Communauté d'Agglomération Nord Basse Terre (Guadeloupe).

En termes méthodologiques, entre six et dix personnes ayant participé à l'élaboration et/ou à la mise en œuvre d'actions inscrites dans le PCET local furent interrogés dans chaque collectivité de manière semi-dirigée à partir d'un guide d'entretien pour une durée moyenne d'une heure. Au total, 66 entretiens furent menés pour 75 personnes (10 entretiens furent menés avec deux personnes) auprès de chargés de mission, chefs de services, directeurs, élus et techniciens, respectant une diversité thématique et sectorielle, organisationnelle, des genres et des tranches d'âge. L'ensemble des enregistrements audio fut retranscrit en verbatim et une analyse qualitative des discours fut effectuée par grille d'interprétation. Ces informations furent complétées par une analyse des actions relevant de l'adaptation (appelées comme telles ou non) inscrites dans chacun des PCET des cas d'études. Les résultats présentés ici réfèrent à la partie de la recherche s'étant attardée à la représentation sociale et à la définition de l'adaptation aux changements climatiques qu'en purent donner les acteurs interrogés et telle qu'elle est ressortie dans les PCET étudiés.

\section{Résultats : l'interprétation de l'adaptation dans les Plans Climat}

L'analyse des dix PCET des cas d'études étudiés montre qu'une action relève de l'adaptation selon l'interprétation qu'en donne la collectivité (Simonet et Leseur, 2015). Ainsi, une action présente dans le volet «adaptation » d'une collectivité peut se retrouver dans un volet « gestion du risque », " anticipation » ou «protection du territoire » dans le PCET d'une autre collectivité voire dans un volet n'ayant aucun lien avec la problématique climatique. À titre d'exemple, l'action « lutter contre la précarité énergétique », présente dans le volet «adaptation » de la ville d'Annecy, se retrouve dans le volet « Réduire l'empreinte écologique de l'habitat et augmenter la performance environnementale des opérations de construction » de la Communauté Urbaine de Dunkerque, dans le volet «Renforcer les solidarités » de Cergy-Pontoise Agglomération ou encore dans le volet "Efficacité énergétique du bâti et politique de l'habitat » de la Communauté d'Agglomération Royan Atlantique. De ce fait, l'action publique territoriale menée en matière d'adaptation aux changements climatiques ne se réduit pas aux actions dénommées «adaptation » mais se révèle de manière «silencieuse » et transversale à travers d'autres actions. Cette transversalité reflète celle avec laquelle les changements climatiques touchent directement ou indirectement l'ensemble des secteurs et thématiques d'une collectivité (urbanisme, gestion des risques naturels, transports, biodiversité, précarité énergétique).

Par ailleurs, les résultats montrent que les communes étudiées intègrent la gestion des risques (naturels, côtiers) dans des volets « adaptation » parce qu'elles disposent de compétences en la matière. À l'inverse, la gestion des risques ne relevant pas de l'échelon intercommunal, les Établissements public de coopération intercommunale (EPCI) ont tendance à appréhender l'adaptation de manière plus large, en l'associant notamment à la sensibilisation aux enjeux climatiques et à la modification des pratiques et des comportements. En dehors de l'interprétation, la recherche montre que les contextes locaux (socioéconomiques, géographiques, patrimoniaux) influencent également la manière de définir une action d'adaptation. Ainsi, les collectivités étudiées expérimentant directement des modifications de leur territoire du fait de tendances marquées (érosion) ou d'événements climatiques extrêmes récurrents (tempêtes) associent davantage l'adaptation à la gestion des risques naturels. A contrario, celles qui n'expérimentent que peu ces manifestations associent davantage l'adaptation à d'autres enjeux locaux (précarité énergétique, végétalisation), manquant d'indicateurs tangibles pour rendre compte des modifications que les évolutions climatiques entraînent sur leur territoire. Sur ce point, il est possible que ce manque puisse être utilisé pour maintenir les intérêts en place et freiner la mobilisation des acteurs locaux autour de la question d'adaptation (Simonet et Leseur, 2015). 


\section{Représentation sociale de l'adaptation auprès des acteurs locaux}

La majorité des entretiens menés témoignent de la difficulté de définir ou de traduire en action la notion d'adaptation aux changements climatiques. De nombreux répondants relatent qu'ils se heurtèrent à cette difficulté dès la phase d'élaboration des PCET. Le manque de définition tranchée de l'adaptation est perçu comme la porte ouverte à une interprétation qui dépend de la sensibilité locale au phénomène climatique et aux enjeux qui y sont rattachés. En règle générale, la dimension abstraite de l'adaptation aux changements climatiques laisse une large interprétation qui rend difficile sa traduction en actions spécifiques, la renvoyant davantage à un cadre d'analyse (Simonet et Leseur, 2015).

Ajouté à cela, plusieurs interlocuteurs pointent l'absence et la difficulté d'associer des indicateurs, quelque chose de comptable ou un produit fini à des actions d'adaptation. Une personne signale que dans la définition même de la mise en œuvre du PCET, il est mentionné de "prendre en compte l'adaptation » sans qu'il n'y ait de précision sur ce qu'il faut y mettre derrière, sur la manière de la chiffrer et tout en n'apparaissant pas obligatoire de le faire. Ce manque d'indicateur est régulièrement comparé avec la métrique utilisée pour mesurer les réductions des émissions de GES telle que la réduction de la facture énergétique. En plus d'être facilement chiffrable, les interlocuteurs précisent que cette mesure est prioritaire compte tenu de l'impact positif sur les financements des collectivités. Certains propos mentionnent que le caractère fluctuant d'une action d'adaptation rend difficile la tâche visant à la cocher " mise en œuvre ", prenant l'exemple de l'initiative intitulée « garantir un soutien aux personnes âgées ».

Par ailleurs, les énoncés discursifs montrent qu'il est perçu que les stratégies d'adaptation relèvent de la réflexion, ne se traduisent pas forcément par des actions et ne dépendent pas nécessairement d'un budget pour exister. Dans ce cas, elles sont davantage associées à quelque chose de théorique et abstrait, qui se réfléchit, s'accommodant bien dans les discours d'ordre général sur la problématique climatique (figure 1). D'autres répondants qualifient l'adaptation de "gros mot dans le sens complexe », établie dans une construction conceptuelle au même titre que le « développement durable ». Quelques propos la relient à une notion scientifique, en vogue dans les milieux de la recherche mais absente du quotidien des collectivités, hormis sur la gestion des risques.

Associant l'adaptation à la gestion des risques, un répondant relate toutefois que le SCOT datant de 2007 de sa collectivité n'utilisait pas l'expression «adaptation », bien que prenant en compte les risques climatiques. En concordance, certains propos confirment que sur le volet littoral, l'expression utilisée demeure la " gestion des risques », sauf si la sémantique est revue après coup. Des propos renseignent sur le fait que le terme " adaptation » a fini par s'imposer tout seul sur des actions élaborées initialement dans l'objectif d'une réponse « à quelque chose de concret ».

L'apposition du terme "adaptation» sur des actions déjà établies est également mentionnée par un répondant lorsque, après avoir mis en place des jardins partagés, l'argumentaire s'est davantage tourné sur la mise en avant de la biodiversité, des îlots de fraicheur, de la convivialité sociale et de l'appropriation de l'espace public. Un autre répondant résume le fait qu'une action, "de toute façon, on a beau l'écrire, on ne la fait pas comme elle est écrite» et "si on a des actions d'adaptation, on les fera comme elles seront acceptées ». Par ailleurs, rajoute un interlocuteur, la prise de distance est nécessaire car «sur ces questions d'adaptation, il faut détacher l'affect».

Concernant l'incertitude liée au futur, les actions d'adaptation sont perçues « un peu à part », notamment parce qu'elles couvrent le moyen et le long terme. Le fait que la notion d'adaptation puisse s'appréhender via un diagnostic des risques et une analyse des impacts potentiels rend compliqué «le fait d'atténuer quelque chose qui n'existerait pas encore». Par ailleurs, plusieurs répondants relient cette idée d'anticipation à la notion d'adaptation, «venue naturellement car le fait de parler d'anticipation, c'est toujours plus positif que l'adaptation», rajoutant qu' «on s'adapte à une contrainte, on n'anticipe pas forcément une contrainte».

Un interlocuteur prend l'exemple de la construction d'un port de plaisance ayant intégré les risques à partir de scénarios climatiques tout en n'étant pas formalisé comme étant de l'adaptation aux changements climatiques, mais comme la transcription d'une sensibilisation locale aux inondations, tsuna- 
mis ou sécheresse. Cette dimension temporelle est également introduite par des propos relatant que les actions d'adaptation ne demeurent pas toujours une fin en soi, du fait de cette difficulté de ne pas savoir précisément à quoi va ressembler le résultat par rapport au projet initial. De ce fait l'adaptation peut davantage prendre la forme d'un cadre d'analyse pour l'ensemble des projets, quels qu'ils soient. Les aspects reliés à la créativité nécessaire pour que «des gens cherchent des solutions » est mentionnée par un répondant, car "celui qui ne s'adapte pas y reste».

Enfin, face à la question de définir l'adaptation aux changements climatiques selon leur propre conception, il existe trois types de réponses. La plupart des acteurs locaux (42\%) associent l'adaptation à la nécessité de sensibiliser les populations, les gestionnaires et les élus aux enjeux climatiques et aux thématiques qui en sont reliées. Cette idée inclut l'association à des modifications de comportements, de mentalités et de pratiques dans l'objectif de diriger la société actuelle vers un mieux vivre en adéquation avec les dynamiques naturelles, le respect du vivant et la bonne gestion des ressources. Cette perspective «naturaliste » se distingue d'un deuxième groupe de réponses $(30 \%)$ qui associent la notion d'adaptation davantage à la gestion des risques par l'aménagement du territoire et du bâti, notamment du fait d'expérimentations passées et actuelles des impacts des changements climatiques sur le territoire de leur collectivité. L'objectif de cette perspective « résiliente » est de protéger le territoire, les populations et les activités contre les aléas des changements climatiques, perspective que l'on retrouve dans la majorité des définitions de l'adaptation issues de la littérature scientifique ou issues des guides pratiques. Sans être significatif, cette catégorie regroupe davantage de collectivités littorales. Les répondants de la dernière perspective $(28 \%)$, appelée «prospective », associent l'adaptation à l'anticipation des changements climatiques par la mobilisation des connaissances et le recours aux outils prospectifs (scénarios, modélisation) afin d'essayer de prévoir les impacts potentiels. Là, l'objectif est de se préparer à des impacts futurs en réduisant leur certitude.

\section{UNE VARIABILITÉ INTERPRÉTATIVE DE L'ADAPTATION AUX MULTIPLES ORIGINES}

Plusieurs facteurs peuvent expliquer la différence entre l'interprétation des acteurs de terrain et les définitions théoriques concernant l'adaptation aux changements climatiques, parmi lesquels l'influence du GIEC sur la littérature scientifique, celle de la sémantique du terme « adaptation » et celle portant sur la manière d'appréhender et penser le « changement ».

\section{Exemples de dires d'acteurs concernant la difficulté d'interprétation de l'adaptation (Simonet et Leseur, 2015)}

"Au départ, ce n'était pas la question de l'adaptation aux changements climatiques. Ça s'est collé après. Au début on répondait à quelque chose de concret. Et par la suite on a intégré toutes les questions environnementales et la notion d'adaptation s'est collée après. [...] Je ne pense pas qu'on ait dit adaptation climatique parce qu'on s'est rendu compte que ça pouvait coller, c'était inconscient, ça s'est imposé tout seul. » E31

"On vient de construire un port de plaisance et on a intégré les risques à partir de scénarios etc. On a intégré ces aspects d'impacts et de conséquences du changement. Ce n'est pas formalisé comme étant des mesures d'adaptation mais dans la réalité on le fait car on a cette sensibilisation aux inondations, tsunamis, sécheresse, etc. » E58

"Contrairement aux autres points du Plan Climat, les aspects d'adaptation au changement climatique ne doivent pas être une fin en soi, ça peut être une grille de questionnement par laquelle passe tous les projets. Par exemple si on a un projet de réaménagement des quartiers, il faut que je pense à plus de nature en ville, les ilots de fraicheur, comment on évite que les gens soient en difficulté énergétique etc. Et puis c'est des questions de réseaux et de matérialisation de matière grise. [...] Il peut vraiment y avoir une particularité dans ce côté doute de ne pas savoir à quoi va ressembler le résultat. On construit un bâtiment à énergie positive ou des jardins, on voit à quoi ça va ressembler. Mais une stratégie autour de ça c'est compliqué. Sur toutes les autres questions, en général on voit la continuité. Mais pas sur les questions d'adaptation. » E2 


\section{L'influence du GIEC sur la construction du terme « adaptation»}

\section{L'origine de la dichotomie mitigation-adaptation}

Publié environ tous les six ans, le tome 2 (qui traite du thème de l'adaptation) du rapport du GIEC a évolué depuis sa première version (1990) aussi bien dans son contenu que dans la diversité disciplinaire des travaux scientifiques pris en compte pour rendre compte de l'état de la problématique climatique. arguant que l'homme s'était toujours adapté au fil des âges lors du premier rapport (1990), l'adaptation n'apparut que lors du deuxième rapport (1995) sous le vocable "adaptibility ». C'est également là que "deux types de réponses pewvent être identifiés : la mitigation et l'adaptation », marquant l'envol de la fameuse dichotomie dans les décennies suivantes (Simonet, 2015). Celle-ci fut justifiée par le fait que la mitigation s'occupe des causes tandis que l'adaptation a pour mission de gérer les conséquences. En établissant un lien direct avec les impacts climatiques, la définition de l'adaptation fut définie à travers les questions relatives à la gestion des risques et des catastrophes naturelles, également discipline de la majorité des responsables de l'élaboration du rapport. Influencée par les quelques recherches de terrain de l'époque (Butzer, 1980; Rosenberg et al., 1989 ; Jodha, 1989), l'adaptation prit la forme d'options techniques à mettre en place pour contenir les éventuels impacts dans les différents secteurs et thématiques. La distinction mitigation-adaptation est à l'origine de conflits entre les tenants de l'une face aux tenants de l'autre, notamment au niveau des négociations internationales. En effet, révélés par l'opposition entre les pays riches (tenants de la mitigation) et les pays pauvres (tenants de l'adaptation), par l'occultation temporaire de l'adaptation de la scène politique ou par une séparation des fonds, les termes n'ont eu de cesse de s'opposer malgré de nombreux partisans de leur intégration. Il faut attendre le quatrième rapport (2007) pour découvrir un chapitre (19) évoquant un chevauchement entre les deux notions.

\section{De l'ancrage dans l'ajustement vers l'émergence de la transformation}

Le vocable "Adaptation» fit son entrée dans le glossaire du deuxième tome du troisième rapport du GIEC (2001). En mentionnant les dimensions socioéconomiques, sa définition se rapproche de celle donnée de la vulnérabilité. Celle-ci détaille notamment les éléments déterminants de la « capacité adaptative » (technologie, ressources économiques, information et aptitudes, infrastructures, institutions et équité) sur laquelle des stratégies peuvent se développer. Néanmoins, bien que présentée sous différentes formes (anticipatrice, réactive, publique, privée, autonome ou planifiée), l'adaptation reste principalement teintée d'« ajustement » (Reghezza et Rufat, 2015). Les liens entre la distribution des ressources, la pauvreté et les pouvoirs ne sont pas discutés. L'adaptation demeure confinée à une liste de conditions techniques pouvant être traitées sans remise en cause de l'ordre social et politique établi (Basset et Fogelman, 2013). La définition de l'adaptation présentée dans le quatrième rapport du GIEC (2007) reprend les mêmes termes qu'en 2001 et reste ainsi vouée à un rôle de réponse palliative. La dimension relative à la «transformation » des systèmes y est occultée et n'apparaît pas comme une option envisageable jusqu'au cinquième rapport (2014) (Basset et Fogelman, 2013). En consacrant une partie entière à l'adaptation, ce dernier opus marque une rupture avec ses précédents. Concrètement, douze nouveaux termes se référant directement à la notion d'adaptation font leur apparition dans son glossaire. La forte tonalité «transformation » des nouvelles acceptions (via notamment l'«adaptation transformationnelle ») témoigne de l'influence des travaux engagées lors des dernières années sur cet aspect de l'adaptation qui continuent d'évoluer au sein d'un nouveau domaine de recherche (Pelling, 2011; O’Brien, 2012; Rickards et Howden, 2012; Park et al., 2012; Eyzaguirre et al., 2014; Simonet, 2015). La définition même de l'adaptation stricto sensu se modifie subtilement via l'ajout de «seeks », laquelle introduit un élément de dynamique $^{2}$. Dès lors, le passage de la vision « ajus-

\footnotetext{
2. Comme nous le verrons plus loin, cet ajout rend la définition plus souple et ne va pas sans rappeler la signification chinoise du terme " adaptation » à travers l'idéogramme (mandarin simplifié) «适应》 (shiying), lequel peut être défini comme "tenter de s'habituer à quelque chose de nouveau » (Simonet, 2008).
} 
tement » vers une perception plus dynamique de l'adaptation permet de mieux prendre en compte la complexité des systèmes et ouvre la porte à la remise en question des pertinences des mécanismes (institutionnels, techniques, financiers) sous-jacents aux systèmes en place. Fait marquant, le glossaire 2014 se pose également en rupture avec la terminologie attenante à la mitigation en présentant deux définitions, une spécifiquement dédiée aux changements climatiques ("Mitigation [of climate change]») et l'autre aux catastrophes naturelles et à la notion de « risque » («Mitigation [of disaster and disaster]»). Cette déclinaison accentue la confusion terminologique générale et rejoint l'ambigüité sémantique déjà soulevée entre « atténuation » et « adaptation » dans la traduction française du glossaire du quatrième rapport (2007) (Simonet, 2011, p. 415). Elle témoigne également des discordes entre disciplines et visions des responsables de l'élaboration des différents chapitres constituant le rapport.

\section{L'influence de l'historique sémantique de la notion d'adaptation}

\section{De la sémantique}

Si on regarde la définition du terme «adaptation » dans le dictionnaire historique de la langue française (Rey, 2006), on sera dans un premier surpris de voir qu'il existe deux sens : le premier en lien avec l'action d'adapter (ou de s'adapter) et le second renvoie à la modification qui en résulte. Autrement dit, l'étymologie du terme « adaptation » fait référence à la fois à un processus (action) et à une finalité (résultat). Au niveau de l'historique sémantique, «adapter » provient du latin apere (lier, attacher), dont le participe passé aptus (apte) ajouté à la locution ad (à, vers) a donné le verbe adaptare (ajuster à, en vue de) (Rey, 2006). Emprunté au latin au XIII e siècle, «adapter» apparut au sens concret (appliquer), puis au figuré (mettre en accord avec quelque chose). Certains usages disparurent (s'adapter contre quelqu'un) et l'emploi actuel de "s'adapter » émergea au XVI ${ }^{\mathrm{e}}$ siècle, accompagné de dérivés (aptitude, approprié ou adhérer) (Rey-Debove et Rey, 2007). «Adaptation» dérive du latin médiéval adaptatio, attesté au XIII ${ }^{\text {e }}$ siècle, mais généralisé en français puis en anglais au XVI ${ }^{\mathrm{e}}$ siècle pour désigner l'action d'adapter au sens d'ajuster. En 1789, «l'ajustement entre deux choses » est la seule définition mentionnée (Cormon, 1789). Au XIX $x^{e}$ siècle, grâce à l'essor de la biologie, l'adaptation inclut l'idée de modification, pour ensuite prendre toute sa dimension sémantique à travers son appropriation transdisciplinaire, notamment en psychologie, et sa transposition en sociologie (Taché, 2003). L'antonyme «inadaptation », apparu en 1931, provient également du latin inaptus, ayant donné «inapte " ou «inapproprié». Il reste utilisé en psychologie, tout comme « désadaptation " (perte de l'adaptation) (1894) et « réadaptation » (1904).

\section{Une fracture épistémologique récente}

La notion d'adaptation s'est tout d'abord construite autour de l'idée générale que la vie avait été « créée » de toutes pièces. Ce présupposé déterministe, ancré notamment par les travaux d'Aristote, fut diffusé à l'échelle européenne pendant de longs siècles, largement repris par la religion judéo-chrétienne. Cette influence participa à sculpter une manière de réfléchir et de percevoir le monde dans laquelle la compréhension de l'environnement se traduit notamment par une séparation entre Nature et Société (Simonet, 2009). Tranquillement logée dans ce déterminisme aristotélicien, l'adaptation révéla sa dimension dynamique au XIX ${ }^{\mathrm{e}}$ siècle, époque de renouveau scientifique qui permit un nouvel essor des connaissances et des réflexions. La biologie participa à une nouvelle manière de concevoir le monde à travers l'idée que les êtres vivants puissent être issus d'une «évolution » permanente et continue. Cette idée révolutionnaire est à l'origine d'une fracture épistémologique dont le mot «adaptation » fut le cœur. D'un déterminisme aristotélicien, la notion d'adaptation acquit une dimension évolutionniste darwinienne. La conception où le monde du vivant « est créé » bascula vers celle où il « se construit » en permanence. Pour l'adaptation, ces découvertes furent le point de départ d'une nouvelle carrière, polysémique celle-ci, à travers une multitude de disciplines reprenant chacune à leur compte les agréments que la notion pouvait offrir. En moins d'un siècle, l'adaptation passa d'un simple terme au rang de concept " effrayant ", comme le nomma Lucien Cuénot (1925), notamment parce qu' « une adaptation est en réalité la solution d'un problème, exactement comme une machine ou un outil fabriqués par l'homme. » 


\section{L’influence des pensées ou comment concevoir le changement}

L'intangibilité des modifications qui surviennent dans les systèmes naturels du fait des changements climatiques participe à la difficulté de saisir la problématique de l'adaptation. Tel que nous le comprenons, la pression anthropique actuelle sur le système climatique se concrétise par une accélération des transformations des milieux naturels et des territoires, déjà en proie à différentes et complexes dynamiques, issues ou non d'autres pressions anthropiques. Pour décrire ces changements en les contextualisant dans les dynamiques continues plus larges qui sont au cœur de la vie, François Jullien utilise très justement le terme de «transformations silencieuses » (Jullien, 2009).

Quel est le poids des tendances engendrées par les changements climatiques sur l'ensemble des transformations silencieuses générales modelant les territoires? Et quelles sont les « adaptations silencieuses », celles qui sans bruit se mettent en place en réponses à ces évolutions subtiles qui touchent aussi bien les milieux naturels, les ressources que la biodiversité? Car face à ces transformations silencieuses qui façonnent les paysages, l'adaptation n'est un qu'un changement en réponse à un changement. C'est ainsi qu'en soi, l'adaptation peut elle aussi être envisagée comme une transformation silencieuse, c'est-à-dire un processus continu par lequel les systèmes s'organisent en fonction de l'évolution de leur environnement.

Cette dimension dynamique est d'ailleurs intégrée dans la définition même du terme « adaptation » et c'est une des principales raisons qui la rend si complexe à saisir. À l'origine de la difficulté de « penser » une « adaptation », il est nécessaire de mieux comprendre le mode de pensée qui a sculpté le terme, issu de la pensée grecque et dont nous sommes empreints. À l'instar de notre langage basé sur des mots précis pour désigner les choses, la pensée grecque privilégie les délimitations. Essayer de dégager une idée claire et la plus précise possible, décliner en formes plus petites et axer le verbe sur l'identité des choses, telles sont quelques-unes des caractéristiques de cette manière d'appréhender le monde (Jullien, 2009).

Pour la pensée grecque dans laquelle la société occidentale a évolué et s'est construite, la fracture épistémologique de l'adaptation est encore extrêmement malaisée à comprendre. En effet, l'outillage conceptuel de la pensée grecque est tellement composé de blocs qu'il devient étroit de penser toute transition entre deux états, comme le sous-entend la dimension dynamique de la notion d'adaptation nouvellement acquise. Or, le passage entre deux états, eux-mêmes ayant de leur stabilité seulement une apparence dans un laps de temps précis, détient une place centrale dans d'autres pensées, telle que la pensée chinoise (dans le sens «tao» du terme). Le mouvement permanent, qui se retrouve dans une communication basée sur des idéogrammes pour nommer les choses, permet d'appréhender le monde comme un jeu de transitions ininterrompues. Et c'est en plongeant dans le fonctionnement d'une telle pensée que la dimension dynamique de l'adaptation se révèle le plus. L'avantage de ce mode de raisonnement est de ne plus s'attarder sur la question du temps, et de celle du commencement et de la fin, et de ce fait sur la question d'un but à atteindre. Elle permet également d'amoindrir l'importance de "l'événement » au profit du processus ayant favorisé le contexte dans lequel il est survenu. Cette mini-révolution épistémologique se répercute aujourd'hui dans le domaine des changements climatiques. Mais entraîne un défi : comment concevoir l'adaptation aux changements climatiques avec une pensée grecque à bords tranchés en difficulté dès lors qu'il s'agit d'estimer un « processus permanent »?

\section{CONCLUSION : COMMENT PENSER L'ADAPTATION?}

De toutes ces considérations épistémologiques et philosophiques, que peut-on déduire pour les impératifs de terrain? En quoi ces connaissances procèdent-elles à fournir une aide à la mise en place d'actions réduisant la vulnérabilité des territoires et des populations face aux impacts des changements climatiques? Enfin, en quoi aident-elles à l'élaboration de stratégies d'adaptation aux changements climatiques telle que l'injonction sur les PCAET le préconise?

Les résultats du projet ABSTRACT-colurba montrent une nouvelle fois les difficultés que rencontrent les acteurs locaux à mettre en place une action collective en matière d'adaptation aux chan- 
gements climatiques à l'échelle d'un territoire. Les changements climatiques s'inscrivant dans une dynamique générale de changements globaux qui touchent l'ensemble de la planète, les territoires sont «sculptés » par de nombreuses autres pressions humaines et sociales, au-delà des événements et des tendances météorologiques. La pression de plus de 7,5 milliards d'humains aux modes de vie toujours plus exigeants en termes de consommation énergétique et de ressources naturelles ne peut désormais plus être «silencieuse » et la gestion des impacts climatiques ne devrait plus être gérée de manière séparée d'autres enjeux inter-reliés. Ainsi, il semble essentiel de garder l'aspect « construit » du terme " adaptation », dont la définition continuera à évoluer au gré des influences scientifiques, disciplinaires et humaines. Ajoutée à cela, la terminologie choisie comporte une charge épistémologique historique importante. «S'adapter aux changements climatiques » demanderait à être précisée dès lors que l'on s'en réfère dans les communications, aussi bien dans les Plans Climat que les communications scientifiques. Car la séparation établie entre « adaptation » et «mitigation », dichotomie construite par les institutions scientifiques, est à interroger : à quel point les actions peuvent se réclamer de l'une ou de l'autre? Et, dans le fonds, est-ce si important que cela? Toute modification, que ce soit dans les systèmes de production, de combustion ou de développement, visant à réduire le volume des émissions de gaz à effet de serre, ne sont-elles pas des adaptations, c'est-à-dire des processus visant à réorganiser ces systèmes pour les faire correspondre à de nouveaux impératifs? Les changements climatiques n'entraînent-ils pas déjà de facto des évolutions de mentalités, des modifications de pratiques ou des changements de comportements, et ce aussi bien dans les manières de faire (construire, aménager, organiser) que dans les manières d'être (conscientisation, réflexions, créativité) ? Et en dépit de la difficulté d'y associer des indicateurs, il reste que ces évolutions silencieuses sont à prendre à compte dans la lutte contre la problématique climatique d'origine anthropique, inscrite elle-même dans une dynamique de changements globaux. De ce fait, l'intégration de la dimension dynamique de l'adaptation entraîne une nouvelle compréhension plus large que l'opérationnalisation d'actions au « coup sur coup » en réaction aux événements extrêmes (par exemple). L'heure semble davantage à l'unification des réponses, en acceptant que leurs délimitations ne soient pas aussi tranchées mais empreints de « gradiance », c'est-à-dire de graduelles nuances. Dans ce cas-ci, la protection (résilience, ajustement, adaptation-état) des systèmes est à différencier de leur transformation (transition, durabilité, adaptation-processus) dans le sens où elle n'est qu'un outil pratique au même titre que la mitigation (réduction des GES) dans la boîte à outils disponibles pour lutter contre les changements climatiques. Cette manière d'organiser les choses, déjà initiée dans plusieurs collectivités en pratique, a l'avantage de rendre l'action publique territoriale en matière climatique plus cohérente et systémique. Cette gradiance entre les deux réponses permet de simplifier les actions à inventer et à mettre en place sans plus se soucier si elles seront "adaptatives » ou «mitigatives », tout en prenant conscience des effets interactives (ou rétroactives) entre elles. De plus, un autre avantage de voir l'adaptation comme telle réside dans l'intégration des politiques climatiques à d'autres politiques territoriales, créant des liens avec les dynamiques sociales notamment. En effet, l'adaptation aux changements climatiques a maintes fois été définie comme un processus social dynamique dans la littérature scientifique, soulevant par ailleurs que la capacité à agir collectivement déterminait l'habileté de la collectivité à mettre en place des actions optimales (Kelly et Adger, 2000). L'intégration peut également embrasser les initiatives locales portées "sans bruit », c'est-à-dire qui ne sont pas portées par une organisation publique ou par un programme, et qui n'en demeurent pas moins des actions mises en place en réponses aux impacts des changements climatiques, nommées dans ce texte les « adaptations silencieuses ». Dès lors, la connexion semble évidente de miser sur un effort de rapprochement des politiques sectorielles mais également sociales et économiques, dans un mouvement davantage systémique au niveau des territoires concernés. Dans la pratique, cette manière de faire impliquerait d'innover au niveau des mécanismes organisationnels afin de tenter une élaboration synergique des différents plans d'aménagement et de gestion des ressources. Pour cela, les barrières institutionnelles liées aux enjeux des différents acteurs et entités en place doivent être mises en lumière puis levées. Enfin, cette manière 
dynamique d'appréhender l'adaptation permet de faire le lien avec « la transition énergétique », tant appelée dans les discours actuels des décideurs publics et des mouvements environnementaux. Vers une société davantage en synergie avec la capacité climatique planétaire sous-entend une capacité à réduire les émissions de gaz à effet de serre en deçà du seuil de leur absorption par la Terre. Cet effort nécessite une période de reconversion des modes de production d'énergie, ceux-là même à l'origine du renforcement de l'effet de serre responsable des changements climatiques actuels. Cette période implique également, qu'on le veuille ou non, de retrouver une adéquation équilibrée entre le nombre d'êtres humains et leur niveau de consommation énergétique adéquat. Et de tels processus relèvent eux aussi d'une « adaptation».

\section{Bibliographie}

Adger W.N., Agrawala S., Mirza M.M.Q., Conde C., O’Brien K., Pulhin J., Pulwarty R., Smit B., et Takahashi K., 2007. Assessment of adaptation practices, options, constraints and capacity, in Climate Change 2007: Impacts, Adaptation and Vulnerability, Contribution of Working Group II to the Fourth Assessment Report of the Intergovernmental Panel on Climate Change, M.L. Parry, O.F. Canziani, J.P. Palutikof, P.J. van der Linden and C.E. Hanson, Eds. Cambridge University Press, Cambridge, UK, p. 717-743.

Ayкut S.C., Dahan A., 2015. Gouverner le climat? 20 ans de négociations internationales. Presses de Sciences Po. 750 p.

Barnett J., 2010. Adapting to climate change: three key challenges for research and policy - an editorial essay. Wiley Interdisciplinary Review of Climate Change, 1, p. 314-317.

Bassett T.J., Fogelman C., 2013. Déjà vu or something new? The adaptation concept in the climate change literature, Geoforum, 48, p. 42-53.

Bertrand F., Larrue C., 2007. Gestion territoriale du changement climatique - Une analyse à partir des politiques régionales. Rapport final, volume 1, synthèse, programme GICC2, Laboratoire Ville-Société-Territoire UMR CITERES 6173, Université de Tours, 64 p.

Bertrand F., Richard E., 2015. La délicate existence locale de l'adaptation aux changements climatiques : avec, sans, ou à côté de l'atténuation, Développement durable et territoires, 6 (3) DOI : 10.4000/developpementdurable.11048.

Biesbroek R., Klostermann J.E.M., Termeer C.J.A.M., Кават P., 2013. On the nature of barriers to climate change, Regional Environmental Change, 13, p. 1119-1129.
Burton I., Huq, S., Lim B., Pilifosova O., Schipper E.L., 2002. From impacts assessments to adaptation priorities: the shaping of adaptation policy, Climate Policy, 2, p. 145159.

Butzer K.W., 1980. Adaptation to global environmental change, Prof. Geogr., 32 (3), p. 269-278.

Cormon F., 1789. Nouveau dictionnaire de sobrino, françois, espagnol et latin, Anvers, Piestre et Delamollière, 3 vol.

Crabbé P., Robin M., 2006. Institutional Adaptation of Water Resource Infrastructures to Climate Change in Eastern Ontario, Climatic Change, vol. 78, p. 103-133.

Cuénot L., 1925. L'adaptation, Paris, Doin, 420 p.

Dessai S., Hulme M., 2004. Does Climate Adaptation Policy need Probabilities?, Climate Policy, 4, p. 107-128.

Eyzaguirre J., Warren F.J., 2014. Adaptation : établir un lien entre la recherche et la pratique, in Warren F.J., Lemmen D.S. (dir.), Vivre avec les changements climatiques au Canada : perspectives des secteurs relatives aux impacts et à l'adaptation, Gouvernement du Canada, Ottawa (Ontario), p. 253-286.

Füssel H.M., 2007. Adaptation planning for climate change: concepts, assessment approaches and key lessons, Sustainability Science, 2, p. 265-275.

Gifford R., 2008. Psychology's essential role in alleviating the impacts of climate change, Canadian Psychology, 49, p. $273-280$.

Grothmann T., Patt A. (2005) Adaptive capacity and human cognition: the process of individual adaptation to climate change, Global Environmental Change, 15(3), p. 199-213.

Huq S., ReID H., 2004. Mainstreaming adaptation in development. IDS Bulletin, 35, p. 15-21.

Jodha N.S., 1989. Potential strategies for adapting to greenhouse warming: perspectives from the developing world, in Rosenberg N.J., Easterling W.E., Crosson P.R., Darmstadter J. (eds), Greenhouse Warming: Abatment and Adaptation, Resources for the Future, Washington DC, p. 147-158.

Jones R.N., Patwardhan A., Cohen S.J., Dessai S., Lammel A., Lempert R.J., Mirza M.M.Q., von Storch H., 2014. Foundations for decision making, in FIELd C.B., Barros V.R., Dokken D.J., Mach K.J., Mastrandrea M.D., Bilir T.E., Chatterjee M., Ebi K.L., Estrada Y.O., Genova R.C., Girma B., Kissel E.S., Levy A.N., MacCracken S., Mastrandrea P.R., White L.L. (eds.), Climate Change 2014: Impacts, Adaptation, and Vulnerability. Part A: Global and Sectoral Aspects, Contribution of Working Group II to the Fifth Assessment Report of the Intergovernmental Panel on Climate Change, Cambridge/New York, Cambridge University Press, p. 195-228.

Jullien F., 2009. Les transformations silencieuses, Paris, Grasset, 200 p.

Kelly P.M., Adger N.W., 2000. Theory and Practice in Assessing Vulnerability to Climate Change and facilitating Adaptation, Climatic Change, 47, p. 325-352.

Lammel A., Dugas E., Guillen Gutierrez E., 2012. L'apport de la psychologie cognitive à l'étude de l'adaptation aux 
changements climatiques : la notion de vulnérabilité cognitive. VertigO, 12 (1) DOI : 10.4000/vertigo.11915.

Leiserowitz A., 2006. Climate change risk perception and policy preferences: the role of affect, imagery, and values, Climatic Change, 77, p. 45-72.

Lorenzoni I., Pidgeon N. F., Nicholson-Cole S., Whitmarsh L., 2007. Barriers perceived to engaging with climate change among the UK public and their policy implications. Global Environmental Change, 17, p. 445-459.

Magnan A., Garnaud B., Billé R., Gemenne F., Hallegatte S., 2009. The future of the Mediterranean from impacts of climate change to adaptation issues, IDDRI, $43 \mathrm{p}$.

Moser S.C., Екsтrom J.A., 2010. A framework to diagnose barriers to climate change adaptation. Proceedings of the National Academy of Sciences, 107, p. 22026-22031.

Naess L.O., Bang G., Eriksen S. Vevatne J., 2005. Institutional adaptation to climate change: Flood responses at the municipal level in Norway, Global Environmental Change, 15, p. 125-138.

O'Brien K., 2012. Global environmental change II. From adaptation to deliberate transformation, Progress in Human Geography, 36, p. 667-676.

Park S.E., Marshall N.A., Jakku E., Dowdd A.M., Howden S.M., Mendham E., Fleming A., 2012. Informing adaptation responses to climate change through theories of transformation, Global Environmental Change, 22, p. 115-126.

Pelling M., 2011. Adaptation to Climate Change: From Resilience to Transformation, Routledge, London, UK, 224 $\mathrm{p}$.

Pigeon P., 2012. Apports de la résilience à la géographie des risques : l'exemple de La Faute-sur-Mer (Vendée, France), VertigO, 12 (1) DOI : 10.4000/vertigo. 12031.

Quenault B., 2013. Retour critique sur la mobilisation du concept de résilience en lien avec l'adaptation des systèmes urbains au changement climatique, EchoGéo, 24 DOI : 10.4000/echogeo.13403.

Reghezza-Zitt M., Rufat S., 2015. L'adaptation en Île-deFrance entre injonction et recyclage. Techniques et politiques de la société de l'incertitude, Développement durable et territoires, 6 (3) DOI : 10.4000/developpementdurable. 11035 .

Reser J.P., Morrissey S.A., Ellul M., 2011. The threat of climate change: psychological response, adaptation, and impacts, in Weissbecker, I. (ed.), Climate change and human well-being: Global challenges and opportunities, New York, NY, Springer Science, p. 19-42.
Rey A., 2006. Dictionnaire historique de la langue française, Paris, Le Robert.

Rey-Debove J., Rey A., 2007. Le Nowveau Petit Robert, Paris, Le Robert.

Richard E., 2013. L'action publique territoriale à l'épreuve de l'adaptation aux changements climatiques: un nouveau référentiel pour penser l'aménagement du territoire? Thèse de doctorat en aménagement de l'espace et urbanisme, Université de Tours, 520 p.

Rickards L., Howden S., 2012. Transformational adaptation: agriculture and climate change, Crop and Pasture Science, 63, p. 240-250.

Rosenberg N.J., Crosson P., Easterling W., Fredericks K., SEejo R., 1989. Policy options for adaptations to climate change, in The Bejer Institute (eds) United Nations Environment Programme, The full range of responses to climate change, p. 69-100.

Simonet G., 2015. Une brève histoire de l'adaptation : l'évolution conceptuelle au fil des rapports du GIEC (1990-2014), Natures, Sciences, Sociétés, 23, p. 52-64.

Simonet G., Leseur A., 2015. Analyse des Barrières et leviers à la mise en place de STRatégies d'Adaptation aux Changements climaTiques, le cas des collectivités urbaines, Rapport final ABSTRACT-colurba, I4CE, ADEME et AFD, 140 p.

Simonet G., 2014. L'adaptation, champ de recherche à part entière? (Editorial), in Adaptation aux changements environnementaux et territoires (numéro thématique sous la direction de Julien Rebotier), Sud-Ouest Européen, 37, p. 13-14.

Simonet G., 2011. Enjeux et dynamiques de la mise en ouvre de stratégies d'adaptation aux changements climatiques en milieu urbain, les cas de Montréal et Paris, Thèse de doctorat en sciences de l'environnement, Université du Québec à Montréal, $486 \mathrm{p}$.

Simonet G., 2008. Le concept d'adaptation : polysémie et implication en sciences de l'environnement, Rapport de synthèse environnementale, Université du Québec à Montréal, Montréal, 31 p.

TACHÉ A., 2003. L'adaptation: un concept sociologique systémique, Paris, L'Harmattan, 362 p.

Van Aalst M.K., Cannon T., Burton I., 2008. Community level adaptation to climate change: the potential role of participatory risk assessment, Global Environmental Change, 18, p. 165-179. 